\title{
ON INTERMEDIATE DIFFERENTIABILITY OF LIPSCHITZ FUNCTIONS ON CERTAIN BANACH SPACES
}

\author{
M. FABIAN AND D. PREISS
}

(Communicated by William J. Davis)

\begin{abstract}
A real-valued function $f$ defined on a Banach space $X$ is said to be intermediately differentiable at $x \in X$ if there is $\xi \in X^{*}$ such that for every $h \in X$ the value $\langle\xi, h\rangle$ lies between the upper and lower derivatives of $f$ at $x$ in the direction $h$. We show that if $Y$ contains a dense continuous linear image of an Asplund space and $X$ is a subspace of $Y$, then every locally Lipschitz function on $X$ is generically intermediately differentiable.
\end{abstract}

Let $(X,\|\cdot\|)$ be a Banach space with dual $X^{*}$ and duality pairing between $X^{*}$ and $X$ denoted by $\langle\cdot, \cdot\rangle$. Recall that the upper and lower derivatives of a function $f: X \rightarrow \mathbb{R}$ at $x \in X$ in a direction $h \in X$ are defined by

$$
D^{+} f(x, h)=\limsup _{t \downarrow 0} \frac{1}{t}[f(x+t h)-f(x)]
$$

and

$$
D_{+} f(x, h)=\liminf _{t \downarrow 0} \frac{1}{t}[f(x, t h)-f(x)]
$$

respectively. The function $f$ is said to be intermediatly differentiable at $x \in X$, with intermediate derivative $\xi \in X^{*}$, if

$$
D^{+} f(x, h) \geq\langle\xi, h\rangle \geq D_{+}(x, h) \quad \text { for all } h \in X .
$$

The aim of this note is to prove the following statement.

Theorem. Suppose that a Banach space $Y$ contains a dense continuous linear image of an Asplund space and that $X$ is a subspace of $Y$.

Then every locally Lipschitz function defined on an open subset $\Omega$ of $X$ is intermediately differentiable at every point of a residual subset of $\Omega$.

The Banach spaces containing a dense continuous linear image of an Asplund space have been extensively studied by Ch. Stegall [11], who calls them $G S G$ spaces. Among other things, he also proved [11, $\$ 4$, Remark 3] that the nonw.c.g. subspace of some $L_{1}(\mu), \mu$ finite, constructed by H. Rosenthal [10] is

Received by the editors April 23, 1990 and, in revised form, July 11, 1990.

1980 Mathematics Subject Classification (1985 Revision). Primary 46G05; Secondary 46B22, $58 \mathrm{C} 20$.

Key words and phrases. Lipschitz function, upper, lower, and intermediate derivative, Asplund space, dentability. 
not GSG. Hence our theorem extends the result of M. Fabian and N. V. Zhivkov [5] who considered the case $X=Y$.

We observe that our result implies the well-known fact [2] that a subspace $X$ of a GSG space is weak Asplund. Indeed, using [15, Proposition 2.1], one easily sees that a stronger statement holds: If a continuous function $f$ defined on an open subset $\Omega$ of $X$ has (one-sided) directional derivative at each point of $\Omega$ and at each direction, then $f$ is generically Gateaux differentiable on $\Omega$.

It does not seem that the proof of the above theorem can be obtained by adapting the proofs from [5, 15]. In fact, a quite different approach works; we use some ideas met in the theory of analytic spaces on one hand and some two-dimensional reasoning similar to those used in $[8,9]$ on other hand.

The crucial concept we use is $d$-A-dentability ${ }^{*}$. Let $E$ be a bounded subset of the dual $X^{*}$ of a Banach space $X$, let $A$ be a bounded absolutely convex subset of $X$; and let $d>0$ be given. We say that $E$ is $d$ - $A$-dentable ${ }^{*}$ if there are $0 \neq e \in X$ and $\gamma>0$ such that the slice

$$
S(E, e, \gamma) \equiv\{\xi \in E:\langle\xi, e\rangle>\sup \langle E, e\rangle-\gamma\}
$$

has $A$-diameter less than $d$; that is,

$$
\operatorname{diam}_{A} S(E, e, \gamma) \equiv \sup \{\langle\xi-\eta, u\rangle: \xi, \eta \in S(E, e, \gamma), u \in A\}<d .
$$

Let us recall that $A \subset X$ is said to have a Stegall property provided that every bounded subset of $X^{*}$ is $d$ - $A$-dentable * for every $d>0$; see [1, pp. 116148], where other equivalent characterizations and other names of this property can also be found.

We also use the notion of a Clarke subdifferential [3]: Given a locally Lipschitz function $f: X \rightarrow \mathbb{R}$ on a Banach space $X$, put

$$
f^{0}(x)(h)=\limsup _{\substack{t \downarrow 0 \\ y \rightarrow x}} \frac{1}{t}[f(y+t h)-f(y)], \quad x, h \in X .
$$

Then the Clarke subdifferential of $f$ at $x \in X$ is defined by

$$
\partial f(x)=\left\{\xi \in X^{*}:\langle\xi, h\rangle \leq f^{0}(x)(h) \text { for all } h \in X\right\} .
$$

Note that $\partial f(x)$ is a nonempty set and that the function $f^{0}(x)(\cdot)$ is subadditive and positively homogeneous for each $x \in X$.

The key lemma we use can be formulated as:

Lemma. Let $G$ be an open subset of a Banach space $X$, consider a Lipschitz function $f: G \rightarrow \mathbb{R}$ with a Lipschitz constant 1 , and put $\partial f(G)=$ $\bigcup\{\partial f(x): x \in G\}$. Let $A$ be an absolutely convex bounded subset of $X$ with the Minkowski's functional $q$. Finally, let $d>0, \gamma>0$, and $e \in X$, with $\|e\|=1$, be such that

$$
\operatorname{diam}_{A} S(\partial f(G), e, \gamma)<d
$$


Then there exist $x$ in $G, \xi$ in the unit ball $B_{X^{*}}$ of $X^{*}$ and $\Delta>0$ such that

$$
|f(x+h)-f(x)-\langle\xi, h\rangle| \leq 3 d q(h)
$$

whenever $h \in X$ and $q(h)<\Delta$.

Proof. Put $\alpha=\sup \{\|u\|: u \in A\}$ and

$$
s=\sup \langle\partial f(G), e\rangle
$$

Choose

$$
\beta \in\left(0, \min \left(\gamma, \frac{d^{2} \gamma}{4 \alpha(d+2 \alpha)}\right)\right)
$$

From the definition of $\partial f(G)$ and using classical facts from real analysis, we can find $x \in G$ such that the limit

$$
V f(x)(e) \equiv \lim _{t \rightarrow 0} \frac{1}{t}[f(x+t e)-f(x)]
$$

exists and $V f(x)(e)>s-\beta$. In fact, find $y \in G$ such that $s-\beta<\sup \langle\partial f(y), e\rangle$; then, according to the definition of $\partial f(y)$, we get $s-\beta<f^{0}(y)(e)$. Also, by the definition of $f^{0}(y)(e)$, find $z \in G$ and $\tau>0$ so that $\{z+t e: t \in[0, \tau]\} \subset G$ and $s-\beta<\frac{1}{\tau}[f(z+\tau e)-f(z)]$. Now the mapping $t \mapsto g(t) \equiv f(z+t e)$, $t \in[0, \tau]$, is Lipschitz. Hence the derivative $g^{\prime}(t)$ exists almost everywhere and moreover,

$$
\int_{0}^{\tau} g^{\prime}(t) d t=f(z+\tau e)-f(z),
$$

which is greater that $\tau(s-\beta)$. It follows that there is $t \in(0, \tau)$ such that $g^{\prime}(t)$ exists and $g^{\prime}(t)>s-\beta$. Then put $x=z+t e$.

Further, from the definition of $\partial f(x)$, we can find $\xi \in \partial f(x)$ such that

$$
V f(x)(e)=\langle\xi, e\rangle \quad(>s-\beta) .
$$

Note that $\xi \in S(\partial f(G), e, \gamma)$ since $\beta<\gamma$. Also there is $\delta \in(0,1)$ such that $u \in G$ whenever $\|u-x\|<2 \delta$ and such that

$$
|f(x+\tau e)-f(x)-\tau\langle\xi, e\rangle| \leq \beta|\tau| \text { for } \tau \in(-\delta, \delta) .
$$

Now fix any $h \in X$ such that $0<q(h)<\min (\delta / \alpha, 2 \beta \delta / d)$, and take

$$
t=d q(h) / 2 \beta \text {. }
$$

Then note that $\|h\| \leq \alpha q(h)<\delta$ and $0<t<(d / 2 \beta) \cdot(2 \beta \delta / d)=\delta$. Let us remark that for $(\tau, \rho) \in[0, t] \times[0,1] \equiv I$ we have

$$
\|x+\tau e+\rho h-x\| \leq \tau+\rho\|h\| \leq t+\|h\|<2 \delta
$$

so $x+\tau e+\rho h \in G$. Thus for $\rho \in[0,1]$, we get

$$
\begin{aligned}
f(x+t e+\rho h) & \geq f(x+t e)-\rho\|h\| \\
& \geq f(x)+t\langle\xi, e\rangle-\beta t-\rho\|h\| \\
& >f(x)+t s-2 \beta t-\|h\|
\end{aligned}
$$


by (5) and (4); and further

$$
f(x+\rho h) \leq f(x)+\|h\| .
$$

And putting the last two inequalities together with $\|h\| \leq \alpha q(h),(6)$, and (3), we get

$$
\begin{aligned}
& f(x+t e+\rho h)-f(x+\rho h)>t s-2 \beta t-2\|h\| \\
& \geq t s-2 \beta t-2 \alpha q(h) \\
&=t s-2 \beta t(1+2 \alpha / d)>t s-d \gamma t / 2 \alpha, \\
& f(x+t e+\rho h)-f(x+\rho h)>t s-d \gamma t /(2 \alpha) \text { for all } \rho \in[0,1] .
\end{aligned}
$$

We now define a mapping $\phi: I \rightarrow \mathbb{R}$ by

$$
\phi(\tau, \rho)=f(x+\tau e+\rho h), \quad(\tau, \rho) \in I .
$$

Note that $\phi$ is Lipschitz and so by the Rademacher theorem, there is a Lebesgue negligible set $N$ in $I$ such that the Gateaux (i.e. Fréchet) derivative $\phi^{\prime}(\tau, \rho)$ exists whenever $(\tau, \rho) \in I \backslash N$. Let us observe that for these $(\tau, \rho)$

$$
\begin{aligned}
& \phi_{1}^{\prime}(\tau, \rho)=V f(x+\tau e+\rho h)(e) \leq s, \\
& \phi_{2}^{\prime}(\tau, \rho)=V f(x+\tau e+\rho h)(h) \geq-\|h\| \geq-\alpha q(h),
\end{aligned}
$$

where $\phi_{1}^{\prime}$ and $\phi_{2}^{\prime}$ mean the partial derivatives of $\phi$ along the first and the second coordinate respectively. Let $\lambda_{1}$ and $\lambda_{2}$ denote the one- and the twodimensional Lebesgue measures respectively.

Consider the following sets:

$$
\begin{aligned}
E & =\left\{(\tau, \rho) \in I \backslash N: \phi_{1}^{\prime}(\tau, \rho)>s-\gamma\right\} ; \\
E_{\tau} & =\{\rho \in[0,1]:(\tau, \rho) \in E\}, \quad \tau \in[0, t] ; \\
E^{\rho} & =\{\tau \in[0, t]:(\tau, \rho) \in E\}, \quad \rho \in[0,1] .
\end{aligned}
$$

Using (7) and (8) we can estimate for almost all $\rho \in[0,1]$,

$$
\begin{gathered}
t s-\frac{d \gamma t}{2 \alpha}<\phi(t, \rho)-\phi(0, \rho)=\int_{0}^{t} \phi_{1}^{\prime}(\tau, \rho) d \tau=\int_{E^{\rho}}+\int_{E^{\rho c}} \\
\leq \lambda_{1}\left(E^{\rho}\right) s+\left(t-\lambda_{1}\left(E^{\rho}\right)\right)(s-\gamma)=\lambda_{1}\left(E^{\rho}\right) \gamma+t s-t \gamma \\
\lambda_{1}\left(E^{\rho}\right)>t-d t /(2 \alpha): \\
\Lambda_{2}(e)=\int_{0}^{1} \lambda_{1}\left(e^{\rho}\right) d \rho>t-d t /(2 \alpha)
\end{gathered}
$$

On the other hand, as $\lambda_{2}(E)=\int_{0}^{t} \lambda_{1}\left(E_{\tau}\right) d \tau$, we can find $\tau \in[0, t]$ such that

$$
\lambda_{1}\left(E_{\tau}\right)>1-d /(2 \alpha)
$$

and that, simultaneously, the derivative $\phi^{\prime}(\tau, \rho)$ exists for almost all $\rho \in$ $[0,1]$. For every such $\rho$ we have $(\tau, \rho) \notin N$ and by the Hahn-Banach theorem, there exists $\eta_{\rho} \in \partial f(x+\tau e+\rho h)$ such that

$$
\left\langle\eta_{\rho}, u\right\rangle=V f(x+\tau e+\rho h)(u)
$$


for all $u$ from the linear span of $e$ and $h$. Therefore, observing that, by (8), $\eta_{\rho} \in S(\partial f(G), e, \gamma)$ for $\rho \in E_{\tau}$, we have by (1) and (9)

$$
\begin{aligned}
|f(x+\tau e+h)-f(x+\tau e)-\langle\xi, h\rangle| & =\left|\int_{0}^{1} \phi_{2}^{\prime}(\tau, \rho) d \rho-\langle\xi, h\rangle\right| \\
& =\left|\int_{0}^{1}\left\langle\eta_{\rho}-\xi, h\right\rangle d \rho\right| \leq\left|\int_{E_{\tau}}\right|+\left|\int_{E_{\tau}^{c}}\right| \\
& \leq \lambda_{1}\left(E_{\tau}\right) d q(h)+\left(1-\lambda_{1}\left(E_{\tau}\right)\right) 2\|h\| \\
& \leq d q(h)+2 d\|h\| /(2 \alpha) \leq 2 d q(h),
\end{aligned}
$$

Finally, note that $x+\tau e, x+\tau e+h \in G$. So we can estimate

$$
\begin{aligned}
f(x+\tau e) & \geq f(x)+\tau\langle\xi, e\rangle-\beta \tau \\
& \geq f(x)+\tau(s-\beta)-\beta \tau \geq f(x)+\tau s-2 \beta t
\end{aligned}
$$

by (5) and (4). Also, there are $\rho_{n} \uparrow 1$ such that

$$
\begin{aligned}
f\left(x+\tau e+\rho_{n} h\right) & =\phi\left(\tau, \rho_{n}\right)=\phi\left(0, \rho_{n}\right)+\int_{0}^{\tau} \phi_{1}^{\prime}\left(\theta, \rho_{n}\right) d \theta \\
& =f\left(x+\rho_{n} h\right)+\int_{0}^{\tau} V f\left(x+\theta e+\rho_{n} h\right)(e) d \theta \\
& \leq f\left(x+\rho_{n} h\right)+\tau s,
\end{aligned}
$$

hence

$$
f(x+\tau e+h) \leq f(x+h)+\tau s
$$

Now by combining the last inequality with (11), we obtain

$$
f(x+h)-f(x)>f(x+\tau e+h)-f(x+\tau e)-2 \beta t .
$$

Thus, putting this inequality together with $(10)$ and $(6)$, we get

$$
f(x+h)-f(x)-\langle\xi, h\rangle>-2 d q(h)-d q(h)=-3 d q(h) .
$$

So far we have been working in the parallelepiped with vertices $x, x+t e$, $x+h$, and $x+t e+h$. Performing an analogous reasoning in the parallelepiped with vertices $x, x-t e, x+h$, and $x-t e+h$ we can find a $\tilde{\tau} \in(0, t)$ such that

$$
|f(x-\tilde{\tau} e+h)-f(x-\tilde{\tau} e)-\langle\xi, h\rangle| \leq 2 d q(h)
$$

and

$$
f(x+h)-f(x)<f(x-\tilde{\tau} e+h)-f(x-\tilde{\tau} e)+2 \beta t .
$$

By combining the last two inequalities and (6) we get

$$
f(x+h)-f(x)-\langle\xi, h\rangle<3 d q(h) .
$$

Proof of Theorem. Let $Z$ an Asplund space and $T: Z \rightarrow Y$ be a linear continuous mapping such that $T(Z)$ is dense in $Y$. We work in the subspace $X$ 
of $Y$. Let $\Omega$ be an open subset of $X$ and $f: \Omega \rightarrow \mathbb{R}$ be a locally Lipschitz function. According to [6, Chapter $1, \S 10]$ we know that a set $M \subset \Omega$ is residual in $\Omega$ if every point $x \in \Omega$ has a neighborhood $W$ such that $M \cap W$ is residual in $W$. It follows that we may assume without loss of generality, that $f$ is (globally) Lipschitz on the whole $\Omega$. Also, we may and do assume that the Lipschitz constant of $f$ is equal to 1 .

For $n, p \in \mathbb{N}$ put

$$
A_{n, p}=\left[n T\left(B_{Z}\right)+\frac{1}{p} B_{Y}\right] \cap X,
$$

where $B_{Z}, B_{Y}$ denote the unit balls in $Z, Y$ respectively. Then we claim that every subset in the unit ball $B_{X^{*}}$ of $X^{*}$ is $3 / p-A_{n, p^{-}}$-dentable ${ }^{*}$. Assuming this fact, then the lemma says that the sets

$$
\begin{array}{r}
M_{n, p}=\left\{x \in \Omega: \exists \xi \in B_{X^{*}} \exists \Delta>0|f(x+h)-f(x)-\langle\xi, h\rangle| \leq(9 / p) q_{n, p}(h)\right. \\
\text { whenever } \left.h \in X \text { and } q_{n, p}(h)<\Delta\right\}
\end{array}
$$

are dense in $\Omega$, where $q_{n, p}$ are the Minkowski's functionals of $A_{n, p}$. Put

$$
\begin{aligned}
\widetilde{M}_{n, p}=\left\{x \in \Omega: \exists \xi \in B_{X^{*}}\right. & \exists r \in(0,1 / p) \\
& \left.\sup \left\{|f(x+r h)-f(x)-\langle\xi, r h\rangle|: h \in A_{n, p}\right\}<10 r / p\right\} .
\end{aligned}
$$

These sets are open because $f$ is Lipschitz. They are also dense in $\Omega$ since they contain $M_{n, p}$. Thus putting $M=\bigcap_{n, p=1}^{\infty} \widetilde{M}_{n, p}$, this set is residual in $\Omega$.

We show that $M$ is the set, which we are looking for. So fix some $x \in M$. For any sequence $\sigma=\left(n_{1}, n_{2}, \ldots\right) \in \mathbb{N}^{\mathbb{N}}$ put $A_{\sigma}=\bigcap_{p=1}^{\infty} A_{n_{p}, p}$. Let us fix for a moment one such $\sigma$. Then for each $p \in \mathbb{N}$ we can find $r_{p} \in(0,1 / p)$ and $\xi_{p} \in B_{X^{*}}$ such that

$$
\left|\frac{1}{r_{p}}\left[f\left(x+r_{p} h\right)-f(x)\right]-\left\langle\xi_{p}, h\right\rangle\right|<\frac{10}{p} \quad \text { whenever } h \in A_{n_{p}, p} .
$$

As the sequence $\left\{\xi_{p}\right\}$ lies in $B_{X^{*}}$, it has a weak ${ }^{*}$ cluster point $\xi_{\sigma}$, say. Fix for a moment any $h \in A_{\sigma}$. For each $i=1,2, \ldots$ there is $p_{i}>i$ such that $\left|\left\langle\xi_{p_{i}}-\xi_{\sigma}, h\right\rangle\right|<1 / i$. Then

$$
\begin{aligned}
\mid \frac{1}{r_{p_{i}}}[ & \left.f\left(x+r_{p_{i}} h\right)-f(x)\right]-\left\langle\xi_{\sigma}, h\right\rangle \mid \\
& \leq\left|\frac{1}{r_{p_{i}}}\left[f\left(x+r_{p_{i}} h\right)-f(x)\right]-\left\langle\xi_{p_{i}}, h\right\rangle\right|+\left|\left\langle\xi_{p_{i}}-\xi_{\sigma}, h\right\rangle\right| \\
& <10 / p_{i}+1 / i<11 / i \rightarrow 0 \text { as } i \rightarrow \infty .
\end{aligned}
$$

And, recalling that $0<r_{p_{i}}<1 / p_{i}<1 / i$, we can conclude that

$$
D^{+} f(x, h) \geq\left\langle\xi_{\sigma}, h\right\rangle \geq D_{+} f(x, h) .
$$


This, of course, holds for every $h \in A_{\sigma}$. Let $\psi_{\sigma}(x)$ denote the set of all the $\xi_{\sigma}$ fulfilling (12). We remark that if $\sigma, \tau \in \mathbb{N}^{\mathbb{N}}$, then there exists $\rho \in \mathbb{N}^{\mathbb{N}}$ such that $A_{\sigma} \cup A_{\tau} \subset A_{\rho}$; hence $\psi_{\rho}(x) \subset \psi_{\sigma}(x) \cap \psi_{\tau}(x)$. And as $\psi_{\sigma}(x)$ are weakly ${ }^{*}$ closed and lie in $B_{X^{*}}$, we get that the intersection

$$
\bigcap\left\{\psi_{\sigma}(x): \sigma \in \mathbb{N}^{\mathbb{N}}\right\}
$$

is nonempty. Take some $\xi$ in this set. Then

$$
D^{+} f(x, h) \geq\langle\xi, h\rangle \geq D_{+} f(x, h) \text { for all } h \in X .
$$

since $\bigcup\left\{A_{\sigma}: \sigma \in \mathbb{N}^{\mathbb{N}}\right\}=X$.

It remains to prove the claim. So fix $n, p \in \mathbb{N}$ and let $E$ be a nonempty subset of $B_{X^{*}}$. Let $\widetilde{E}$ denote the weakly ${ }^{*}$ closed convex hull of $E$. Following the proof of [7, Theorem 12], let $F$ be a weakly ${ }^{*}$ closed convex subset of $B_{Y^{*}}$, minimal with respect to the inclusion, and such that

$$
\widetilde{E}=\{\xi / X: \xi \in F\},
$$

where $\xi / X$ means the restriction of $\xi$ to the space $X$. As $Z$ is Asplund, there is $z \in Z$ and $\beta>0$ such that the slice $S\left(T^{*}(F), z, \beta\right)$ is nonempty and

$$
\operatorname{diam}_{B_{Z}} S\left(T^{*}(F), z, \beta\right)<1 /(n p) \text {. }
$$

Then $S(F, T z, \beta) \neq \varnothing$ and we estimate

$$
\begin{aligned}
& \operatorname{diam}_{n T\left(B_{Z}\right)} S(F, T z, \beta)=\sup \left\{\left\langle\xi_{1}-\xi_{2}, v\right\rangle: \xi_{i} \in S(F, T z, \beta), v \in n T\left(B_{Z}\right)\right\} \\
& \quad=\sup \left\{\left\langle T^{*} \xi_{1}-T^{*} \xi_{2}, u\right\rangle: T^{*} \xi_{i} \in S\left(T^{*}(F), z, \beta\right), u \in n B_{Z}\right\} \\
& \quad=n \operatorname{diam}_{B_{Z}} S\left(T^{*}(F), z, \beta\right)<n /(n p)=1 / p .
\end{aligned}
$$

Now the set

$$
E_{1}=\{\xi / X: \xi \in F \backslash S(F, T z, \beta)\}
$$

is weakly ${ }^{*}$ closed, convex, and, by the minimality, $E_{1} \neq \widetilde{E}$. So there are $0 \neq e \in X$ and $\alpha>0$ such that $S(\widetilde{E}, e, \alpha) \neq \varnothing$ and

$$
S(\widetilde{E}, e, \alpha) \cap E_{1}=\varnothing .
$$

Then $\xi \in S(F, T z, \beta)$ whenever $\xi \in F$ and $\xi / X \in S(\widetilde{E}, e, \alpha)$. Hence

$$
\begin{aligned}
\operatorname{diam}_{A_{n, p}} S(E, e, \alpha) \\
\quad \leq \operatorname{diam}_{A_{n, p}} S(\widetilde{E}, e, \alpha) \\
\quad=\sup \left\{\left\langle\eta_{1}-\eta_{2}, v\right\rangle: \eta_{i} \in S(\widetilde{E}, e, \alpha), v \in\left[n T\left(B_{Z}\right)+\frac{1}{p} B_{Y}\right] \cap X\right\} \\
\quad \leq \sup \left\{\left\langle\xi_{1}-\xi_{2}, v\right\rangle: \xi_{i} \in S(F, T z, \beta), v \in n T\left(B_{Z}\right)+\frac{1}{p} B_{Y}\right\} \\
\quad \leq \sup \left\{\left\langle\xi_{1}-\xi_{2}, v\right\rangle: \xi_{i} \in S(F, T z, \beta), v \in n T\left(B_{Z}\right)\right\}+2 / p \\
\quad=\operatorname{diam}_{n T\left(B_{Z}\right)} S(F, T z, \beta)+2 / p<3 / p .
\end{aligned}
$$

Finally we recall that $\widetilde{E}$ is a weakly ${ }^{*}$ closed convex hull of $E$, so $S(\widetilde{E}, e, \alpha) \neq$ $\varnothing$ implies that $S(E, e, \alpha)$ is also nonempty. This finishes the proof of the claim. 


\section{REFERENCES}

1. R. Bourgin, Geometric aspects of convex sets with the Randon-Nikodym property, Lecture Notes in Math., vol. 993, Springer-Verlag, Berlin, 1984.

2. J. P. R. Christensen and P. Kenderov, Dense strong continuity of mappings and the RandonNikodym property, Math. Scand. 54 (1984), 70-78.

3. F. H. Clarke, Generalized gradients and its applications, Trans. Amer. Math. Soc. 205 (1975), 247-262.

4. J. Diestel and J. J. Uhl, Jr., Vector measures, Math. Surveys No. 15, Amer. Math. Soc., Providence, R. I., 1978.

5. M. Fabian and N. V. Zhivkov, A characterization of Asplund spaces with the help of local E-supports of Ekeland and Lebourg, C. R. Acad. Bulgare Sci. 38 (1985), 671-674.

6. K. Kuratowski, Topology, Vol. I, New York, 1966.

7. I. Namioka and R. R. Phelps, Banach spaces which are Asplund spaces, Duke Math. J. 42 (1975), 735-750.

8. D. Preiss, Gateaux differentiable functions are somewhere Fréchet differentiable, Rend. Circ. Mat. Palermo, (2) 33 (1984), 123-133.

9. _ Differentiability of Lipschitz functions on Banach spaces, J. Funct. Anal. 91 (1990), 312-345.

10. H. Rosenthal, The heredity problem for weakly compactly generated Banach spaces, Compositio Math. 28 (1974), 83-111.

11. Ch. Stegall, The Radon-Nikodym property in conjugate Banach spaces II, Trans. Amer. Math. Soc. 264 (1981), 507-519.

12. M. Talagrand, Espaces de Banach faiblement K-analytiques, Ann. of Math. (2) 110 (1979), 407-438.

13. L. Vašák, On one generalization of weakly compactly generated Banach spaces, Studia Math. 70 (1981), 11-19.

14. N. V. Zhivkov, Generic Gateaux differentiability of locally Lipschitzian functions, Constructive Function Theory 1981, Sofia, 1983, pp. 590-594.

15. _ Generic Gateaux differentiability of directionally differentiable mappings, Rev. Roumaine Math. Pures Appl. 32 (1987), 179-188.

Sibeliova 49, 16200 Prague 6, Czechoslovakia

Department of Mathematics, University College London, Grower Street, London WC1E 6BT ENGLAND 